\title{
Supernovae and their Progenitors
}

\author{
F.-K. Thielemann, Garching *
}

(Max - Planck - Institut für Astrophysik)

Supernovae are explosive stellar events which release typically of the order $10^{51}$ erg in kinetic energy and electromagnetic radiation. Their visual luminosity is comparable to the light emission of an entire galaxy, containing $10^{11}$ stars. This brightness decreases by an order of magnitude within the first two months. In the explosion, matter is ejected with velocities of the order of 10000 $\mathrm{km} / \mathrm{s}$, expanding into interstellar space and forming a so-called supernova remnant. The interaction of the supernova with its environment, into which it is expanding leads to a variety of phenomena observable in radio, optical, ultraviolet, and $\mathrm{X}$-ray wavelengths. The remnants created by the historically known supernovae in our Galaxy, e.g. Supernova 1006, SN 1054 (Crab Nebula), SN 1572 (Tycho's SN), and SN 1604 (Kepler's $\mathrm{SN})$, where the number indicates the year of appearance, can still be observed. Fig. 1 shows a radio image of $\mathrm{SN}$ 1604 at a wavelength of $21 \mathrm{~cm}$.

Matter, ejected by supernovae into the interstellar medium was processed by nuclear reactions during stellar evolution and is the main source of intermediate and heavy atomic nuclei in galactic evolution. Stars which formed later will incorporate this material and will have a larger enrichment in metals (the astronomical term for elements heavier than helium). This recycling process explains a continuous increase in metallicities towards the present value. During the birth of the Universe (the Big Bang) only isotopes of hydrogen, helium, and lithium were produced. Whilst a first generation of very massive stars might have produced $10^{-3}$ of the present metallicities, from then on, supernovae have been responsible for the metal enrichment.

Present observational techniques make it possible to observe supernova outbursts in distant galaxies, typically

\section{*}

Presently at the Department of Astronomy, University of Illinois, Urbana
10 events per year. One can divide SNevents into two major classes, called type I and type II. SN of type II are observed in spiral arms of spiral galaxies (active regions of ongoing star formation) which can be understood if the progenitors are massive fast evolving stars. Their optical spectra are characterized by hydrogen lines. The light curve (optical luminosity as a function of elapsed time after the outburst) shows a plateau for several months after an initial peak and decline. Those features are explained in terms of an extended envelope of solar composition, which has not been processed by nuclear reactions.

The distribution of $\mathrm{SN}$ of type I is not limited to spiral arms within spiral galaxies. Elliptical galaxies, many of which have no ongoing star formation, show also and only SN I events. This favours old stellar populations (white dwarfs) as possible progenitors which are triggered by a delayed mechanism to undergo a supernova explosion. The optical spectra of these $\mathrm{SN}$ are rich in lines of heavy elements, the light curves show an exponential decay. The latter can be explained if unstable radioactive nuclei, produced in the explosion, decay over several months. Then decay energy is still released in the expansion phase. There is a strong indication for the decay chain of ${ }^{56} \mathrm{Ni}\left({ }^{56} \mathrm{Ni} \rightarrow{ }^{56} \mathrm{Co} \rightarrow{ }^{56} \mathrm{Fe}\right)$ with a production of roughly $0.5 M_{0}$ (solar masses) per event.

SN I remnants show no central neutron stars, i.e. objects of the order of 1.4 $M_{0}$ with densities of nuclear matter, supporting the fact that $\mathrm{SN}$ I cause a complete disruption of the progenitor star. Some remnants of SN II, the most prominent one being the Crab nebula, contain pulsars. These are point-like sources of pulsed radio, optical on X-ray emission. They are interpreted as rotating neutron stars with high magnetic fields ( $10^{12}$ Gauss). A more detailed study of the mechanisms, responsible for SN I and SN II events, requires a detour through the theory of stellar evolution.

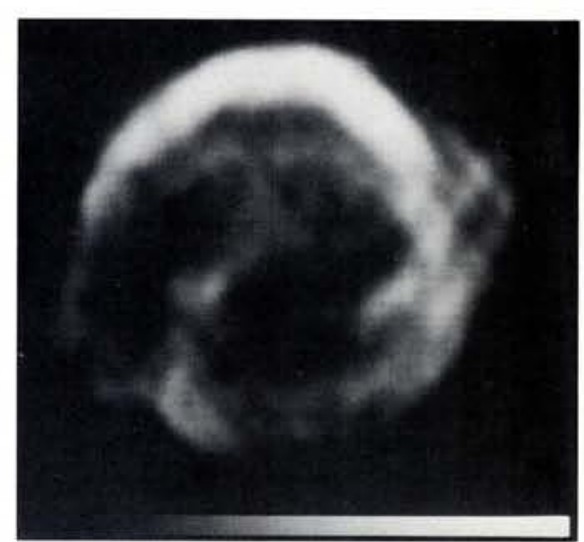

Fig. 1 - Radio continuum image of Kepler's supernova remnant taken with the VLA Nery Large Array) at a wavelength of $21 \mathrm{~cm}$ (Matsui et al. Ap.J. (1984) 287, 295. The diameter of the remnant is $4.4 p c$ and the distance approximately $5 \mathrm{kpc}\left(1.3 \times 10^{14} \mathrm{~km}\right.$ and $1.5 \times 10^{17} \mathrm{~km}$, respectively).

\section{Stellar Evolution}

The reader might be referred to Iben and Renzini (1984) and references therein. The driving force of stellar evolution is gravitational contraction, which is halted only by internal pressure which can counterbalance the gravitational forces. Normally this pressure (radiation pressure, pressure of the electron gas and the atomic nuclei) is a function of temperature. Energy losses by radiation can be balanced by contraction (gain of potential energy) or by the fusion energy of atomic nuclei. A star goes through a sequence of stable burning phases where thermal pressure is provided by the energy gain due to fusion reactions, with intermediate contraction periods, leading to higher central temperatures. Increasing average velocities or the kinetic energy of atomic nuclei, obeying Boltzmann statistics, enable fusion reactions to take place between heavier nuclei with larger electric charge.

The burning stages are:

(1) Hydrogen burning $\left(1-4 \times 10^{7} \mathrm{~K}\right)$

(2) Helium burning $\left(1-3 \times 10^{8} \mathrm{~K}\right)$

(3) Carbon burning $\left(6-10 \times 10^{8} \mathrm{~K}\right)$

(4) Neon burning $\left(1.2-1.3 \times 10^{9} \mathrm{~K}\right)$

(5) Oxygen burning $\left(1.5-2.0 \times 10^{9} \mathrm{~K}\right)$

(6) Silicon burning: photodisintegrations of ${ }^{28} \mathrm{Si}$ above $3 \times 10^{9} \mathrm{~K}$ produce $\alpha$ particles, protons and neutrons. Generally all reaction links by both strong and electromagnetic reactions are now open and a thermodynamic equilibrium of nuclear abundances is approached. Nuclei with the highest binding energies per nucleon (iron group) are produced.

Each of these burning stages proceeds first in the central core; subsequently it moves outward to form a shell burning source and the star eventually forms an onion skin structure. After Si 
burning, the nuclear abundances are in a thermodynamic equilibrium (nuclear statistical equilibrium $=$ NSE) and further temperature increase cannot cause another sequence of fusion reactions. The result is that the iron core of such an evolved star contracts and collapses. The events that follow will be discussed later.

In the hydrogen and helium burning stages cooling is done very inefficiently through radiation transport. Photons emitted in the burning core undergo a large number of scattering events until they are emitted from the photosphere of the star. This leads to large burning cores and long durations of their burning stages $\left(t_{\mathrm{HB}} \cong 10^{10}\left(\mathrm{M} / \mathrm{M}_{\mathrm{o}}\right)^{-3} \mathrm{y}\right.$ is the average time scale for hydrogen burning). After helium burning, at higher densities, a much more powerful mechanism for energy loss becomes available. Neutrino-antineutrino pairs are produced by electron-positron pair anihilation, by electron-photon scattering processes and by electron plasma oscillations (plasmon neutrinos). As those neutrinos can escape freely, burning cores are smaller and the energy loss mechanism is most efficient.

With increasing densities in late burning stages, the electron gas shows signs of partial degeneracy, and its pressure becomes the most important part of the total pressure. The pressure of a degenerate gas is a function of density only and Chandrasekhar showed that an object is then stable against contraction when its mass is less than $1.46\left(2 Y_{\mathrm{e}}\right)^{2} M_{\mathrm{o}} \cdot Y_{\mathrm{e}}$ is the electron abundance or number of electrons per nucleon, being 0.5 for nuclei with an equal number of neutrons and protons.

Applying this to stellar evolution we see that the sequence of contraction and ignition phases set out above holds true only if the core masses exceed the Chandrasekhar limit. Evolution calculations show that this is true only for stars with initial masses larger than $10 M_{0}$. They go through all the central burning stages until an iron core develops. Very massive ones undergo so strong a mass loss by stellar winds that they loose their extended envelopes of unburned hydrogen and have a helium composition at their surface (Wolf-Rayet stars).

Stars with initial masses in the range 8 $\leq M / M_{0} \leq 10$ develop a highly (electron) degenerate core before oxygen ignition. Then the equation of state [pressure as a function of temperature, density, and composition; $\left.p=p\left(T, \rho, Y_{i}\right)\right]$ becomes independent of temperature. Oxygen ignition, energy release, and temperature increase do not result in a rise of pressure. Such a situation leads to a thermal runaway until the temperature is high enough partially to lift the degeneracy. This fixes the evolution, and a combined oxygen and silicon burning gives again a contracting iron core as final result.

For $M \leq 8 M_{\circ}$ evolution calculations show that after core helium burning, the outward moving hydrogen and helium burning shells come very close together. This proximity causes instabilities which lead to thermal pulses and drive strong stellar winds. These wind losses involve the entire material outside the carbonoxygen core and leave a bare core with a mass below the Chandrasekhar limit. This core turns into a white dwarf star which undergoes no further nuclear evolution.

\section{Type II Supernovae}

The final evolutionary states of massive stars $\left(M \geq 8 M_{0}\right)$ have an inner core of iron and iron group nuclei (Fe, $\mathrm{Mn}, \mathrm{Co}$, $\mathrm{Ni}$ ). The transformation of $\mathrm{Si}$ to $\mathrm{Fe}$ at temperatures of 3 to $3.5 \times 10^{9} \mathrm{~K}$ and densities of the order $10^{8} \mathrm{~g} / \mathrm{cm}^{3}$ lasts about one day. The time scales for burning stages from hydrogen to silicon burning are logarithmically decreasing!

When the iron core size passes the Chandrasekhar mass $M_{\mathrm{Ch}}$ an additional contraction phase starts: during contraction the Fermi energy of the electron gas grows and at $\rho=10^{9} \mathrm{~g} / \mathrm{cm}^{3}$ it reaches $5 \mathrm{MeV}$. At such electron energies the endoergic reaction of electron captures on stable nuclei (inverse $\beta^{-}$-decay) is allowed. A result is the reduction of the electron abundance $Y_{\mathrm{e}}$ and the pressure of the degenerate electron gas. This speeds up the contraction which develops into a collapse. Another effect is that increasing temperatures towards $10^{10} \mathrm{~K}$ also force a partial break-up of iron nuclei into free particles - neutrons, protons and $\alpha$-particles. This is again an endoergic process which reduces the internal energy, resulting in a further speed up of the collapse. The production of free protons makes electron capture on protons dominant. The reduction of the electron abundance $Y_{\mathrm{e}}$ causes a decrease of the Chandrasekhar mass $M_{\mathrm{Ch}}$, which sets the size of the homologously collapsing central core. While $Y_{\mathrm{e}}$ has a value of 0.50 in the early burning phases, electron captures in silicon burning change it already to 0.46 . At densities of $4 \times 10^{11} \mathrm{~g} / \mathrm{cm}^{3}$ it is down to $0.35-0.39$.

At these densities, neutrinos (formed by the mechanisms discussed above and electron captures) can no longer escape freely. They are trapped inside a radius which can be described as the "neutrinosphere", similar to the photosphere in a star. Weak interactions come into an equilibrium, as the reverse reactions (i.e. neutrino captures) also become possible. The collapse is only halted when nuclear densities are reached at 2 to $3 \times 10^{14} \mathrm{~g} / \mathrm{cm}^{3}$. A transition occurs to a giant nucleus of the size of the homologously collapsed core. Nuclear matter is highly incompressible, i.e. additional compression leads to a strong rise in pressure. The inner core stops collapsing, becomes firm like a hard golf ball, infalling material rebounds, and an outward moving shock wave is initiated. The traditional picture is that this shock wave lifts off the mantle, reaches the outer envelope within days and causes an observable SN II event, with the inner core left behind in the form of a neutron star.

The difficulty with this scheme is that the final size of the homologously collapsing core is substantially smaller than the original iron core when the contraction started. This is due to the quadratic dependence of $M_{C h}$ on $Y_{e}$ which decreases during the collapse phase. The outward shock wave originates at the edge of the homologous core, travels through matter consisting of iron nuclei, heats up this material, causes dissociation of iron nuclei into nucleons, and thus looses kinetic energy. If the difference between the original iron core and the homologous core is too large, the shock dies and no ejection of the mantle occurs. The critical mass of the initial iron core for a successful core-bounce mechanism seems to lie between 1.25 and $1.35 \mathrm{M}_{\text {。 }}$. These are only found for stars with relatively low masses. Hillebrandt (1984), however reports a successful explosion for a 9 and a $10 M_{0}$ star.

The collapse phase of the central core is of the order of seconds. The propagation of the shock wave through the iron mantle lasts about $0.03 \mathrm{~s}$. If the shock wave is stalled there is hope for other mechanisms which work on longer time scales. Bethe and Wilson (1985) continued calculations over one second after the core bounce. They found that after time scales related to neutrino diffusion out of the inner core $(0.2-0.5 \mathrm{~s})$, neutrino absorption and emission processes on nuclei in the shock region heat up material, increase the pressure and revive the stalled shock wave. Although the energies found are somewhat too small for typical SN II events, it sheds light on the subject. The region close to the neutrino sphere is unstable against turbulent convection which would deposit material with trapped neutrinos at loca- 
tions outside the neutrino sphere. At the present, no calculations have included this effect, but it is expected to be more efficient than neutrino diffusion (Arnett 1985).

Both the neutrino diffusion and convection mechanisms, however, work on similar time scales. These are longer than in the pure core bounce and infalling matter increases the central "proto" neutron star by roughly $0.3 M_{0}$. With present uncertainties of the equation of state, the limiting mass of a degenerate nucleon gas which is stable against contraction, varies between 1.5 and $2.0 \mathrm{M}$ 。 $\left(M_{C h}\right.$ for nucleons). This leaves the possibility that massive stars can undergo a successful SN II event, according to the delayed explosion mechanism, and end with a central object which eventually turns into a black hole, rather than a neutron star.

The outward moving shock wave travels through the adjacent zones and initiates phases of explosive silicon, oxygen, and neon burning. The stellar zones of carbon, helium, and hydrogen burning are essentially unaltered. The final composition in the inner part of a $25 M_{0}$ star can be seen in Fig. 2, where the composition is shown as a function of radial mass (rather than radius). Outer zones would include products of helium and hydrogen burning. The unprocessed hydrogen-rich envelope starts at $M(r)=$ $9 M_{0}$. This result was obtained when a $\mathrm{SN}$ II explosion was simulated by introducing an outward velocity of mass zones at $\cong 1.48 M$ with a total kinetic energy of $1.1 \times 10^{51} \mathrm{erg}$. The uncertainty in the mass cut between the ejected mantle and the inner core affects especially the amount of iron in the ejecta.

\section{Type I Supernovae}

The lack of hydrogen (and also helium) in $\mathrm{SN}$ I spectra indicates that bare carbon-oxygen cores are involved in those explosions. Bare $\mathrm{C}-\mathrm{O}$ cores are the final stages of intermediate mass stars $(M \leq$ $8 M_{0}$ ) which loose their outer hydrogen and helium shells by strong stellar winds and form a "planetary nebula" and a white dwarf, consisting of helium burning products, i.e. carbon and oxygen.

What mechanism can lead to the explosive ignition of white dwarfs? About $50 \%$ of all stars are members of binary stellar systems. If the two stars have different masses, their evolutionary times will be different. While the more massive star might already be in its final stage and have become a white dwarf, the other member can still be in the stage of core hydrogen burning. Undergoing shell hydrogen and core helium burning it

\section{NATO Advanced Study Institute on Physically-Based Modelling and Simulation of Climate and Climatic Change 11-23 May 1986 in Erice, Sicily}

The primary objective of the course is to develop understanding of the design, validation and application of physically-based climate models in the simulation and understanding of past, present and potential future climates.

The lecturers will be A. Arakawa, E. Barron, L. Bengtsson, W. Bourke, K. Bryan, Y. Fouquart, L. Gates, K. Hasselmann, K. Laval, M. MacCracken, Y. Mintz, J. Mitchell, G. North, A. Oort, G. Philander, V. Ramanathan, B. Saltzman, A. Semtner, G. Sommeria, $H$. Sundqvist, M. Tiedtke and T. Wigley.

Persons with an interest in climate modelling and simulation, including advanced graduate students, recent Ph.D.s, and research assistants, who wish to attend the ASI as a student should send a curriculum vitae and two references to the Director of the ASI

Professor Michael E. Schlesinger

Department of Atmospheric Sciences

Oregon State University

Corvallis, Oregon 97331 USA

by December 1985. Limited travel money is available to support students. Applicants should indicate their requirements. turns into a red giant and the size of its envelope expands tremendously. If both stars form a close system, the common gravitational potential can favour mass overflow from the outer zones of the extended envelope (the red giant fills its "Roche-lobe").

Mass transfer rates in excess of $4 \times$ $10^{-8} M_{0} \mathrm{y}^{-1}$ result in almost steady hydrogen and helium burning during the ac- cretion onto the white dwarf. This enlarges the $\mathrm{C}-\mathrm{O}$ core until $M_{\mathrm{Ch}}$ is approached and a contraction and central ignition of carbon sets in under degenerate conditions. Such an event leads to the complete disruption of the white dwarf and can explain a SN I explosion. But in general a whole set of scenarios is possible, including direct accretion of helium or even carbon and oxygen in a close dou-
Fig. 2 - Composition of the inner $2.5 \mathrm{M}_{\mathrm{o}}$ of $25 \mathrm{M}_{\mathrm{o}}$ star (Woosley and Weaver 1982) after explosive nuclear processing in the supernova shock front. In a SN II event the outer part will be ejected into the interstellar medium, the inner (dashed) part can form a neutron star. The original $\mathrm{He}$ and $\mathrm{H}$-burning zones are located at $6.5 \mathrm{M}_{\mathrm{o}}$ and $\mathrm{M}_{\mathrm{O}}$, respectively.

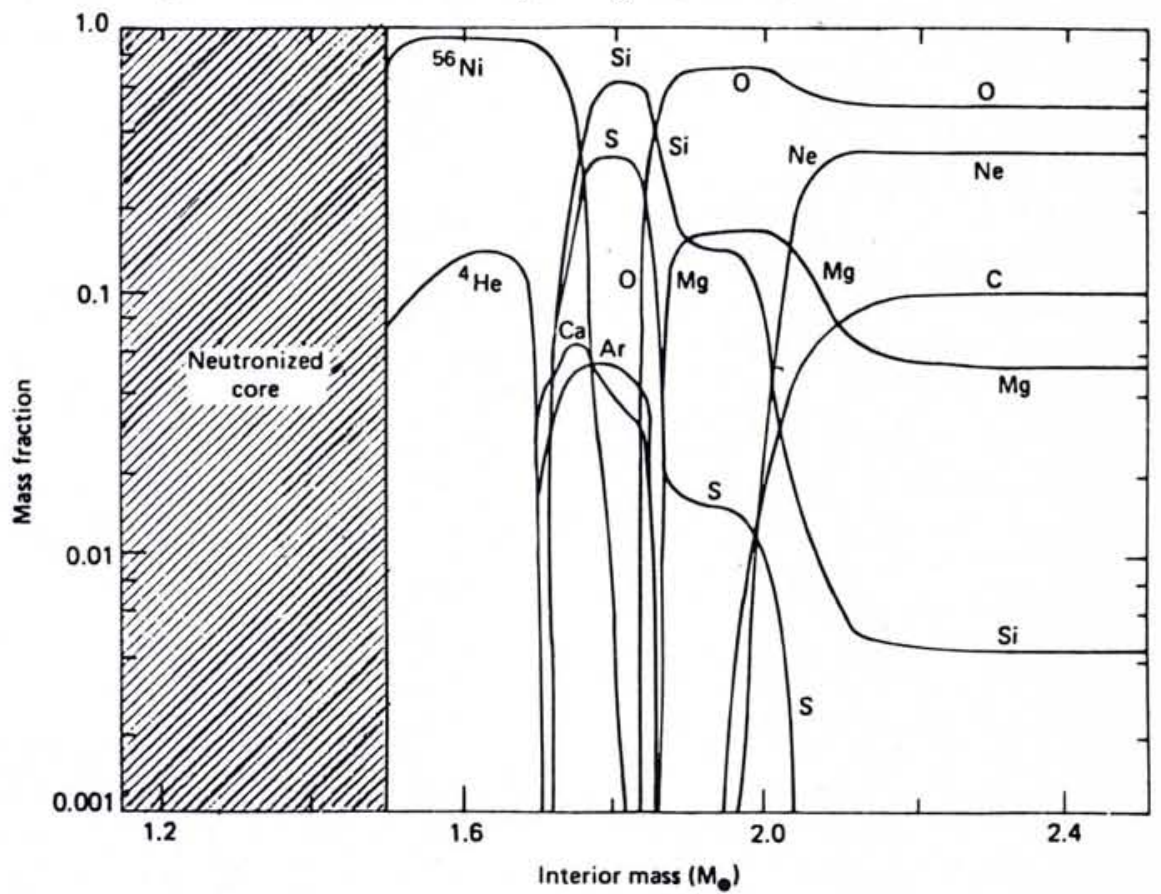




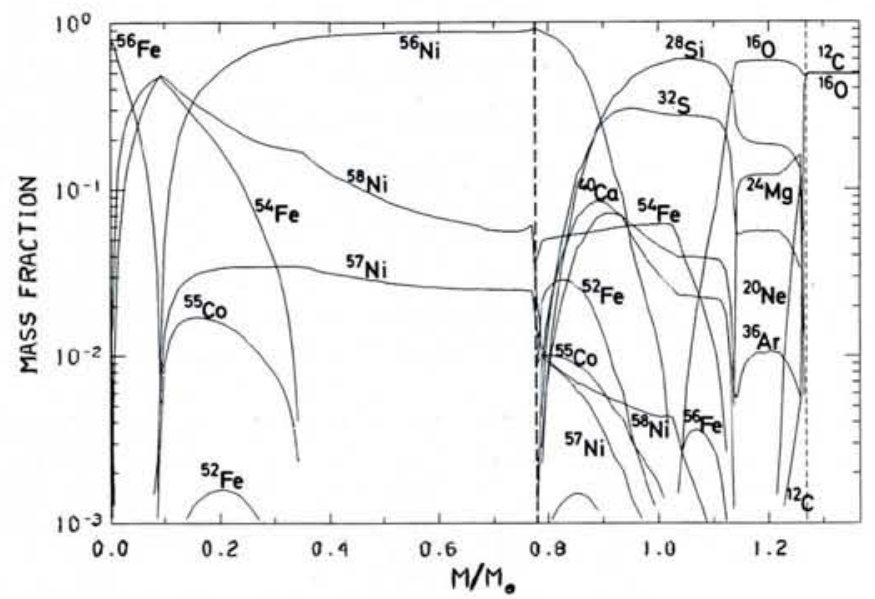

Fig. 3-Composition for a carbon deflagration model of a type / supernova. The inner $1.23 \mathrm{M}_{O}$ of the original $\mathrm{C}-\mathrm{O}$ white dwarf underwent nuclear processing in the explosion. The star is completely disrupted and $0.58 \mathrm{M}_{\mathrm{O}}$ of ${ }^{56} \mathrm{Ni}$ are produced (Nomoto, Thielemann, Yokoi 1984).

ble white dwarf system. For a complete review see Iben and Tutukov (1985). High accretion rates of $\mathrm{C}$ and $\mathrm{O}$ in close double white dwarf systems $(\geq 2 \times$ $10^{-6} M_{0} y^{-1}$ ) can, however, ignite carbon off-centre, burn non-explosively, and follow the evolution of originally more massive stars.

If the helium accretion rate is less than $4 \times 10^{-8} M_{0} y^{-1}$, a helium layer is formed on top of the C-O white dwarf. With increasing size it will reach the point where helium ignites in a flash under the given degenerate conditions. Depending upon the strength of the flash, either a single outward detonation wave (through the He-layer) or a double detonation wave (also proceeding into the $\mathrm{C}$ $\mathrm{O}$ core) forms. Such waves will transform the fuel into nuclear statistical equilibrium (NSE), leaving only iron group nuclei.

As discussed before, ignition under degenerate conditions always leads to a thermal runaway, because the pressure is only a function of density. The strength of the runaway determines if it ends in an explosion or a controllable event. The strong temperature dependence of the triple-alpha rate in $\mathrm{He}$ burning (transformation of helium into carbon via unstable ${ }^{8} \mathrm{Be}$ ) and the energy release cause a very strong runaway and a detonation wave. Degenerate carbon ignition gives rise to a weaker runaway and a deflagration wave. The main characteristics of deflagration and detonation are the following:

(a) in a detonation, a strong shock wave is formed and peak temperatures in the shock are sufficient for explosive ignition of the fuel. A detonation wave moves supersonically when compared with the sound speed in matter in front of the approaching shock wave.

(b) in the deflagration, the compression is not sufficient to cause high enough peak temperatures to ignite the fuel and the burning front propagates via mixing of burning and unburned material behind the compresssion wave. Thus, the burning front moves subsonically and allows for the expansion of matter before the approaching burning front.

Central carbon ignition in a $\mathrm{C}-\mathrm{O}$ white dwarf results in a deflagration wave. The expansion of matter before the burning front leads to a weakening of the burning front. While high temperatures are obtained at the centre of the white dwarf and nuclear reactions allow the build up of iron group nuclei, the outer regions where the burning front is already weakened considerably, produce intermediate nuclei like $\mathrm{Si}, \mathrm{S}, \mathrm{Ar}$, and $\mathrm{Ca}$. $\mathrm{A}$ detonation wave would incinerate the whole white dwarf into iron group nuclei, contrary to what is observed in the spectra of SN I: Fig. 3 shows the radial composition of the products of explosive processing in a deflagration model. In the inner core $0.58 \mathrm{M}_{\mathrm{o}}$ of ${ }^{56} \mathrm{Ni}$ is formed; the energy of the explosion is $1.3 \times$ $10^{51} \mathrm{erg}$. The outer zones show products of incomplete silicon, and explosive oxygen, neon, and carbon burning. $\mathrm{SN} \mathrm{I}$ are the dominant iron contributors to galactic evolution. At present it should be noted that carbon deflagration supernovae are in accordance with roughly $90 \%$ of the observed $\mathrm{SN}$ I events; another class called peculiar SN I still awaits explanation. The general evolution of binary systems certainly deserves further study.

\section{Observations}

Optical observations of SN II events give information mainly about the progenitor star. The plateau in the light curve and the presence of hydrogen lines indicate an extended unprocessed envelope. Unfortunately those observations give no clue to the explosion mechanism or nucleosynthesis in the deeper layers. Such formation could only be obtained from SN II events, if the neutrino pulse, resulting from the collapse and core bounce, could be detected and compared with model predictions. So far information only comes from supernova remnants which expose all of their ejected matter and (if existing) also a central pulsar (neutron star). The best known one is the Crab pulsar but it should be noted that, at present, only five of 30 supernova remnants in an $\mathrm{X}$-ray survey show point sources (Helfand and Becker 1984). This sample includes SN I and SN II remnants. Thus, there is only evidence for a neutron star in $1 / 3$ of the SN II events if $\mathrm{SN} \mathrm{I}$ and SN II are equally present in the given sample. Whether this indicates a specific value of rotation and intrinsic magnetic field, which would not allow us to see these neutron stars, or if it gives evidence for black hole formation is still in question.

The Crab nebula, containing a pulsar and no strong enhancements of elements heavier than sulphur, is in accordance with a SN II explosion of a relatively low mass ( $\cong 10 M_{0}$ ) progenitor. Those are expected to eject only minor amounts of heavy elements. Strangely enough, however, a Ni (and no $\mathrm{Fe}$ ) overabundance is observed.

Cassiopeia $A$ is a supernova remnant with an age of approximately $340 \mathrm{y}$. No historical reports recorded that event, supporting the fact that it was optically subluminous. This indicates the absence of an extended hydrogen envelope, supporting the interpretation of a massive progenitor, which lost its envelope during prior evolution. The enhanced $\mathrm{O}, \mathrm{Si}, \mathrm{S}, \mathrm{Ar}$, and $\mathrm{Ca}$ abundances and the absence of a pulsar (neutron star) are also in favour of this interpretation. Small amounts of Fe suggest that the mass cut between the ejected mantle and the remaining core was at a relatively large radius (black hole?). This is an interpretation, relying on a massive progenitor and that a central black hole will not be observed. Further investigations are needed, however, especially constraints on the mass of the remnant.

The absence of overlying hydrogen and helium shells exposes the products of explosive nucleosynthesis shortly after a SN I event. Fig. 4 shows the optical spectrum of SN 1981b with lines of $\mathrm{O}, \mathrm{Mg}, \mathrm{Si}, \mathrm{S}, \mathrm{Ca}$, and $\mathrm{Co}$. For comparison a "synthetic" spectrum is shown, calculated with the abundances of Fig. 3 and the hydrodynamic behaviour of the corresponding model (Branch et al. 1985). 


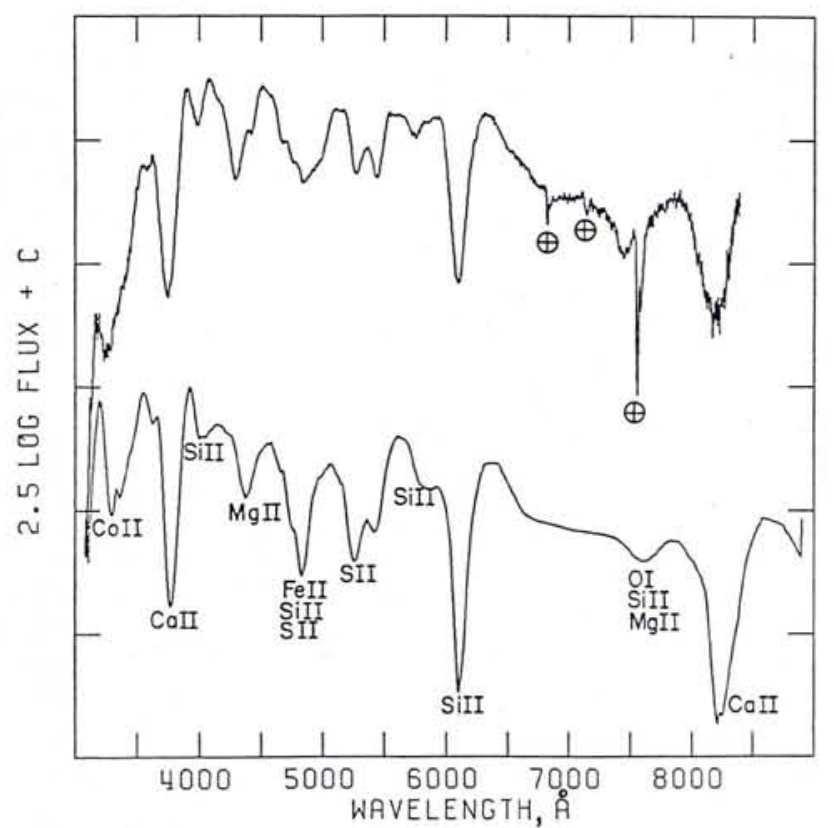

The spectrum was taken 32 days after the explosion. At later times $(\cong 100$ days), the interior, which originally contained large amounts of ${ }^{56} \mathrm{Ni}$, becomes visible. SN 1972e shows a feature at late times, decaying with the half-life of ${ }^{56} \mathrm{Co}$, which can be identified with a Colll line (Woosley, Axelrod, Weaver 1984). This also supports the production of radioactive ${ }^{56} \mathrm{Ni}$, which decays to ${ }^{56} \mathrm{Co}(6.1 \mathrm{~d})$ and ${ }^{56} \mathrm{Fe}(78.76 \mathrm{~d})$. The exponential decay of the supernova light curve requires an amount of roughly 0.5 $\mathrm{M}_{\circ}$ of ${ }^{56} \mathrm{Ni}$.

Extensive studies of supernova remnants have been undertaken with the $X$ ray satellites HEAO 2 (Einstein Observatory), Tenma (Japan) and EXOSAT

\section{REFERENCES}

Arnett W.D., Proceedings of the Fifth Moriond Astrophysics Meeting, eds. J. Audouze and J. Tran Thanh Van (Reidel, Dordrecht), 1985.

Bethe H.A. and Wilson J.R., Ap. J. (1985) 295 , in press.

Branch D., Doggett J., Nomoto K. and Thielemann F.-K., Ap. J. (1985) in press.

Helfand D.J. and Becker R.H., Nature $\mathbf{3 0 7}$ (1984) 215.

Hillebrandt W., Ann. New York Acad. Sci. 422, (1984) 197 (11th Texas Symposium on Relativistic Astrophysics).

Iben I.Jr. and Renzini A., Phys. Rep. 105 (1984) 329.

Iben I.Jr. and Tutukov A.V., Ap. J. Suppl. (1985) 58.

Nomoto K., Thielemann F.-K. and Yokoi K., Ap. J. (1984) 286,644.

Woosley S.E., Axelrod T.S. and Weaver T.A., Stellar Nucleosynthesis, eds. C. Chiosi and A. Renzini (Reidel, Dordrecht) 1984.

Woosley S.E., and Weaver T.A., Essays in Nuclear Astrophysics, eds. C.A. Barnes, D.D. Clayton and D.N. Schramm (Cambridge University Press) 1982, p.377. tified.
Fig. 4 - Observational spectrum of SN 1981b (upper curve). Features identified with a sign are due to terrestrial absorption. The lower curve shows a "synthetic" spectrum calculated with the abundances and the hydrodynamical input from a carbon deflagration model (Branch et al. 1985), shifted for ease of comparison.

(ESA, Europe). Two of the best studied objects are the historical remnants Tycho (SN 1572) and Kepler (SN 1604). No pulsar was observed, supporting models which predict the complete disruption of the progenitor. Strong emission lines of $\mathrm{Si}, \mathrm{S}, \mathrm{Ar}$, and $\mathrm{Ca}$ are observed. The most recent analysis of Tenma data for the Tycho remnant results in ratios between the $\mathrm{Si}, \mathrm{S}, \mathrm{Ar}$, and $\mathrm{Ca}$ abundances which are roughly solar, while $\mathrm{Fe}$ is about a factor of two overabundant. The abundances shown in Fig. 3 give solar ratios for $\mathrm{Si}$ to $\mathrm{Ca}$ and an $\mathrm{Fe}$ overabundance slightly in excess of two. This seems to give additional support to an interpretation based on the carbon deflagration model of SN I.

\section{UNIVERSITY OF} MANCHESTER

\section{Department of Physics}

Postdoctoral Research Associate in Experimental Nuclear Structure Physics

Applications are invited for the above SERC-funded post, tenable for the period October 1st, 1985 to January 31st, 1988. (Post may possibly be extended for a further period.) The successful candidate will be expected to initiate and assist with research at the Nuclear Structure Facility, a $20 \mathrm{MV}$ tandem van de Graaff which is now operational at the Daresbury Laboratory. Applicants are expected to hold a Ph.D. degree in Nuclear Structure Physics and should have an aptitude for pursuing research in experimental physics. Salary will be on the Research Associate (IA) scale, with placement depending on age (e.g. 27 year old with Ph.D. = point 4 on scale $=£ 8,920$ p.a.). Superannuation. Applications, with full C.V. and names of two referees to:

Dr. R. Chapman,

Department of Physics,

The University,

Manchester, M13 9PL from whom further details may be obtained.

\section{THE PENNSYLVANIA STATE UNIVERSITY}

\section{Theoretical Condensed Matter Physics and Statistical Mechanics}

The Department of Physics is seeking candidates for a tenure-track position of Assistant or Associate Professor of theoretical condensed matter physics and statistical mechanics starting in the 1985-86 or 1986-87 academic year. Candidates should have an established record of research accomplishments and may expect to work in conjunction with the ongoing research effort at University Park. Minimal requirements include a Ph.D. degree in this field and some postdoctoral experience. A desire and aptitude for the teaching of undergraduate and graduate students is essential.

Send application, including a curriculum vitae and names of at least four referees, to:

Gerald A. Smith, Head, Department of Physics,

Box Z, 104 Davey Laboratory,

University Park, PA 16802 USA,

by October 15, 1985 or until a suitable pool of applicants is iden- 